\title{
Evaluation of non-Rutherford Alpha Elastic Scattering Cross-sections for Silicon
}

\author{
A.F Gurbich ${ }^{1, *}$ and C. Jeynes ${ }^{1}$ \\ ${ }^{1}$ University of Surrey Ion Beam Centre, Guildford GU2 7XH, England
}

(Dated: June 25, 2013)

\begin{abstract}
The experimental data available for $(\alpha, \alpha)$ silicon elastic scattering cross section at angles and energies suitable for Ion Beam Analysis have been reviewed. The spectra of alphas elastically scattered from a thick uniform silicon target were measured with a small step over the entire energy range studied. The analysis of the data was made in the framework of the R-matrix theory, with potential scattering being taken into account using optical model. The obtained results provide the evaluated differential cross sections for silicon $(\alpha, \alpha)$ elastic scattering in the energy range from Rutherford scattering up to $5.2 \mathrm{MeV}$.
\end{abstract}

\section{INTRODUCTION}

Ion Beam Analysis (IBA) is a common and powerful thin film depth profiling technique [1]. IBA exploits the interactions of rapid charged particles with matter to determine the composition and structure of the surface regions of solids, the differential cross-section data being needed to derive element concentrations through computer simulation of measured spectra. The analysis of silicon samples by IBA methods is widely used in numerous laboratories. Since silicon wafers constitute matrices on which semiconductor structures are built, even where the analysis of silicon itself is not important to the materials characterisation problem, the presence of silicon as a matrix element will preclude application of IBA methods unless the cross-section for the reaction employed is known in detail. There are a number of benefits in use for IBA of ${ }^{4} \mathrm{He}$ elastic backscattering at elevated energies where the elastic scattering cross-section is non-Rutherford and consequently has to be determined through measurements and evaluation.

Evaluation is a powerful approach that can produce recommended cross-sections for the interaction of low energy charged particle with nuclei. The results achieved so far are summarized in [2]. The evaluation methodology applied in the present work resembled a standard approach in all respects save one. Generally established steps starting from a compilation of relevant experimental data followed by their examination and critical selection were made. The specific feature of the procedure employed was adjusting of free nuclear model parameters to fit both the available differential cross-section data and thick target yields measured with a uniform silicon target.

\footnotetext{
* Corresponding author (on leave from Institute for Physics and Power Engineering, Obninsk 249033, Russia): gurbich@ippe.ru
}

Such an approach made it possible to adjust resonance parameters in the cases when the cross-section structure was measured with insufficient energy resolution.

\section{REVIEW OF THE AVAILABLE DATA}

The differential alpha elastic scattering cross sections for silicon at low energy were found in six papers [38]. The excitation functions were obtained in the energy range from 3.6 to $5.8 \mathrm{MeV}$ for 19 different angles from $173^{\circ}$ down to $82^{\circ}$ in [3], at angles from $130^{\circ}$ to $170^{\circ}$ with $10^{\circ}$ step in the energy interval of 3.1 to $7.7 \mathrm{MeV}$ in [4], and at four angles $\left(168^{\circ}, 126^{\circ}, 89^{\circ}, 70^{\circ}\right)$ in the energy region of $4.9-11.7 \mathrm{MeV}$ in [5]. For the work [6] the data are available only for $165^{\circ}$ in the energy range from 2.5 to $6.0 \mathrm{MeV}$. Also in [7-8] the data were measured for only one angle $\left(170^{\circ}\right.$ in [7] and $172^{\circ}$ in [8]) in the energy range of 2.0-9.0 and 3.8-4.6 MeV respectively. Natural silicon $\left(92.23 \%\right.$ of ${ }^{28} \mathrm{Si}, 4.67 \%$ of ${ }^{29} \mathrm{Si}$, and $3.10 \%$ of $\left.{ }^{30} \mathrm{Si}\right)$ was used for manufacturing targets in all the cases. The accuracy of the measured data was reported to be $3-7 \%$.

The measurements carried out in all the works but one ([3]) were performed with thin targets. In [3] the crosssection was derived from the spectra of alphas elastically scattered from a thick target. It should be noted that the energy step in thin target measurements significantly exceeded a typical width of the resonances specific for the case studied and the resonance structure was smeared due to energy straggling in the thick target measurements. The work [3] is the only one where the resonance parameters for $\alpha+{ }^{28} \mathrm{Si}$ were derived from the experimental data. These parameters are listed in the compilation [9]. A striking discrepancy between corresponding energy level parameters of ${ }^{32} \mathrm{~S}$ is observed when compilations [9] and [10] are compared. 


\section{MEASUREMENTS}

The measurements were done using a 2 MV Tandetron of Surrey University Ion Beam Centre. Two surface barrier detectors were located at scattering angles of $149.2^{\circ}$ and $172.8^{\circ}$ determined using a beam line laser. The solid angles subtended by the detectors were 3.50 and $1.25 \mathrm{msr}$ respectively. Electronics calibration was made with an $\mathrm{Au} / \mathrm{Ni} / \mathrm{SiO}_{2} / \mathrm{Si}$ sample as described in [11]. Beam current was $40 \mathrm{nA}$ with a nominal size of the beam spot on the target of $1 \mathrm{~mm}$. An amorphised Si sample was used, the amorphisation being achieved with a multiple energy ${ }^{28} \mathrm{Si}$ implantation up to $2 \mathrm{MeV}$ on a $\mathrm{LN}_{2}$ cooled stage.

The sharp ${ }^{12} \mathrm{C}(\alpha, \alpha){ }^{12} \mathrm{C}$ resonance at $4262 \mathrm{keV}$ and ${ }^{16} \mathrm{O}(\alpha, \alpha){ }^{16} \mathrm{O}$ resonance at $3038 \mathrm{keV}$ were used to verify the machine energy calibration. The absolute beam energy uncertainty was estimated to be about $4 \mathrm{keV}$. Totally 97 spectra were measured in the energy interval of 3.7-6.1 MeV.

\section{CALCULATIONS}

The R-matrix theory was employed for calculations of the ${ }^{28} \mathrm{Si}(\alpha, \alpha)^{28} \mathrm{Si}$ cross sections. In order to take into account tails of broad single particle resonances the phases obtained in the framework of the optical model with Saxon-Woods real potential well and a surface absorption were taken instead of hard sphere ones routinely used in the R-matrix theory. Explicit formulas can be found elsewhere [12]. The cross-section for natural silicon was calculated as a weighted sum of the cross-sections of its three stable isotopes according to their relative abundance. As far as minor silicon isotopes produce only small contribution to the sum the cross-sections for them were assumed to be Rutherfordian.

The spectra of backscattered alphas were calculated with account of all the broadening effects. For a given projectile energy the corresponding depth $x$ where the energy of the slowing down particle reaches energy $E$ was calculated for each of the $d \sigma(E) / d \Omega$ values, stopping power for alphas in silicon being taken from [13]. Then a convolution of $d \sigma(E) / d \Omega$ with a function representing energy spreading was made. Bohr's theory was assumed for energy straggling. Another convolution was applied in order to take into account energy spreading for the outgoing particle and the detector resolution.

\section{RESULTS AND DISCUSSION}

The comparison of the evaluated cross-section with the available experimental data in the vicinity of $170^{\circ}$ is presented in Fig. 1 whereas the comparison of the measured and simulated spectra is shown in Fig. 2. As is seen from the figures the theoretical results are in a good agreement with experiment. The resonance parameters used in the calculations are listed in Table 1. These parameters sig- nificantly differ from [3]. An attempt to reproduce the cross-sections and spectra using parameters obtained in [3] gave unsatisfactory results in both cases. It is strange enough that the elastic alpha width in [3] constitutes only small fraction of the total width for the majority of the resonances. Except for $(\alpha, \gamma)$ with normally very small width the only competitive channel is ${ }^{28} \mathrm{Si}(\alpha, \mathrm{p}){ }^{31} \mathrm{P}$ $(\mathrm{Q}=-1.916 \mathrm{MeV})$, however calculations with parameters from [3] produce unrealistically large cross-section for this channel and the corresponding protons are not observed in the measured spectra.

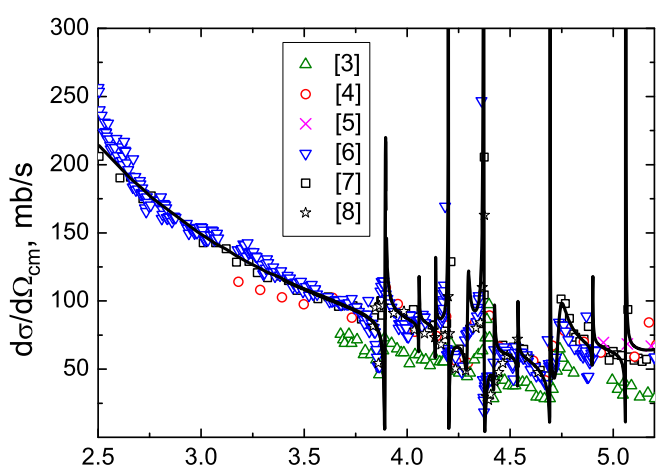

FIG. 1. The evaluated cross sections and the available experimental data for alpha elastic scattering from silicon at $\sim 170^{\circ}$.

TABLE I. Resonance parameters for ${ }^{28} \mathrm{Si}(\alpha, \alpha){ }^{28} \mathrm{Si}$.

\begin{tabular}{c|c|c}
\hline \hline$E_{l a b}, \mathrm{MeV}$ & $J^{\pi}$ & $\Gamma_{l a b}, \mathrm{keV}$ \\
\hline 3.876 & $1^{-}$ & 3.6 \\
4.059 & $0^{+}$ & 1.7 \\
4.139 & $0^{+}$ & 1.2 \\
4.200 & $3^{-}$ & 1.3 \\
4.309 & $0^{+}$ & 8.9 \\
4.381 & $3^{-}$ & 3.3 \\
4.430 & $0^{+}$ & 4.7 \\
4.540 & $0^{+}$ & 1.9 \\
4.693 & $5^{-}$ & 0.6 \\
4.821 & $0^{+}$ & 67.0 \\
4.900 & $2^{+}$ & 2.1 \\
5.069 & $5^{-}$ & 0.6 \\
\hline \hline
\end{tabular}

\section{CONCLUSIONS}

The analysis of the present work revealed significant problems with the available information on the resonance structure observed in the ${ }^{28} \mathrm{Si}(\alpha, \alpha){ }^{28} \mathrm{Si}$ scattering. The evaluated cross section can be calculated for any scattering angle by the on-line SigmaCalc calculator at http://www.surreyibc.ac.uk/sigmacalc/. 

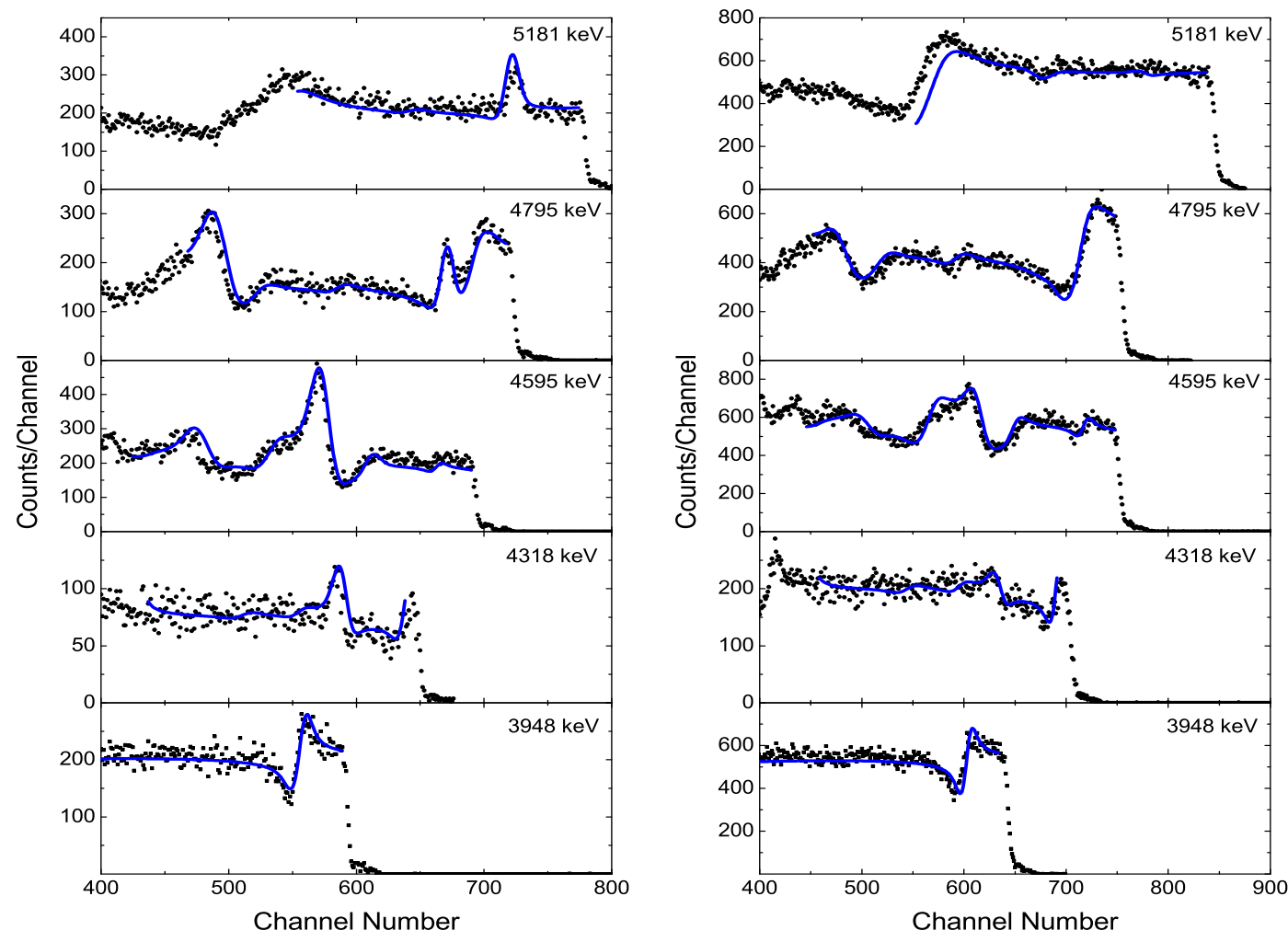

FIG. 2. Comparison of measured and calculated spectra for scattering angles of $172.8^{\circ}$ (left) and $149.2^{\circ}$ (right).

AFG is grateful to the International Atomic Energy Agency for supporting the work and to the Leverhulme Trust for funding his temporary position at Surrey University. CJ is grateful to EPSRC (UK) for support of the measurements under contract number GR/R50097/01.
This work has been supported by the European Community as an Integrating Activity Support of Public and Industrial Research Using Ion Beam Technology (SPIRIT) under EC contract no. 227012. Science And Tech. 4, 41 (2011). 
1703 (2010).

[3] K.-M. Kallman, Zeit. Phys. A 356, 287 (1996).

[4] A. Coban, F.Z. Khiari, M.S. Abdelmonem, A. Aksoy, A.A. Naqvi, Nucl. Phys. A 678, 3 (2000).

[5] J.J. Lawrie, A.A. Cowley, D.M. Whittal, S.J. Mills, W.R. McMurray, Zeit. Phys. A 325, 175 (1986).

[6] M.K. Leung, Ph.D. Dissertation, Univ. OF Kentucky (1972).

[7] H.-S. Cheng, H. Shen, F. Yang, J.-Y. Tang, Nucl. Inst. Meth. Phys. Res. B 85, 47 (1994).

[8] R. Somatri, J.F. Chailan, A.Chevarier, N.Chevarier et al.,
Nucl. Inst. Meth. Phys. Res. B 113, 284 (1996).

[9] C. Ouellet, B. Singh, Nucl. Data Sheets 112, 2199 (2011).

[10] P.M. Endt, Nucl. Phys. A 521, 1 (1990).

[11] C. Jeynes, N.P. Barradas, M.J. Blewett, R.P. Webb, Nucl. Inst. Meth. Phys. Res. B 136-138, 1229 (1998).

[12] A.F. Gurbich, Nucl. Inst. Meth. Phys. Res. B 266, 1193 (2008).

[13] D. Niemann, G. Konac, S. Kalbitzer, Nucl. Inst. Meth. Phys. Res. B 118, 11 (1996). 